This item was submitted to Loughborough's Research Repository by the author.

Items in Figshare are protected by copyright, with all rights reserved, unless otherwise indicated.

\title{
Trace formulae and high energy asymptotics for Stark operator
}

PLEASE CITE THE PUBLISHED VERSION

LICENCE

CC BY-NC-ND 4.0

\section{REPOSITORY RECORD}

Korotyaev, E., and A. Pushnitski. 2019. "Trace Formulae and High Energy Asymptotics for Stark Operator". figshare. https://hdl.handle.net/2134/446. 


\title{
Trace formulae and high energy asymptotics for Stark operator
}

\author{
E. Korotyaev* and A. Pushnitski ${ }^{\dagger}$
}

February 2002

\begin{abstract}
In $L^{2}\left(\mathbb{R}^{3}\right)$, we consider the unperturbed Stark operator $H_{0}$ (i.e., the Schrödinger operator with a linear potential) and its perturbation $H=H_{0}+V$ by an infinitely smooth compactly supported potential $V$. The large energy asymptotic expansion for the modified perturbation determinant for the pair $\left(H_{0}, H\right)$ is obtained and explicit formulae for the coefficients in this expansion are given. By a standard procedure, this expansion yields trace formulae of the Buslaev-Faddeev type.
\end{abstract}

\section{Introduction and Main Results}

1. The Stark operator. In $L^{2}\left(\mathbb{R}^{3}, d \mathbf{x}\right)$ we consider the unperturbed and perturbed Stark operators

$$
H_{0}=-\Delta+\langle\boldsymbol{\beta}, \mathbf{x}\rangle \text { and } H=H_{0}+V \text { with } V=\bar{V} \in C_{0}^{\infty}\left(\mathbb{R}^{3}\right),
$$

where $\boldsymbol{\beta} \in \mathbb{R}^{3}, \boldsymbol{\beta} \neq 0$ is a constant vector (the strength of the external homogeneous electric field) and $\langle\cdot, \cdot\rangle$ is the inner product in $\mathbb{R}^{3}$. The operators $H_{0}$ and $H$ are self-adjoint on the same domain and $C_{0}^{\infty}\left(\mathbb{R}^{3}\right)$ is a core for both $H_{0}$ and $H$. The spectrum of both $H_{0}$ and $H$ is purely absolutely continuous and coincides with $(-\infty, \infty)$ (see $[1,9])$.

2. Notation. We denote $\mathbb{C}_{+}=\{z \in \mathbb{C} \mid \operatorname{Im} z>0\}$. The variable $\lambda$ has the meaning of a spectral parameter and varies over $\mathbb{R}$, whereas the variable $z$ varies over $\mathbb{C}_{+}$. By $\mathfrak{S}_{1}$ and $\mathfrak{S}_{2}$ we denote respectively the trace class and the Hilbert-Schmidt class of compact operators. We use the standard notation for the Heaviside function: $\theta(t)=(|t|+t) / 2$. We denote by $C$ various constants such that their value is not important for our construction.

3. The modified perturbation determinant. The main objects of the present paper are the modified perturbation determinant $\Delta_{H / H_{0}}(z)$, the spectral shift function $\xi(\lambda)$ for the pair (1.1), and the harmonic conjugate $h(\lambda)$ of $\xi(\lambda)$. We start by defining the modified perturbation determinant:

$$
\Delta_{H / H_{0}}(z)=\operatorname{det}\left(\frac{H-z}{H+i} \frac{H_{0}+i}{H_{0}-z}\right), \quad z \in \mathbb{C}_{+} .
$$

Lemma 1.1. (i) For any $z \in \mathbb{C}_{+}$,

$$
(H-z)(H+i)^{-1}\left(H_{0}+i\right)\left(H_{0}-z\right)^{-1}-I=(z+i)(H+i)^{-1} V\left(H_{0}-z\right)^{-1} \in \mathfrak{S}_{1} .
$$

${ }^{*}$ Institut für Mathematik, Humboldt Universität zu Berlin, Rudower Chaussee 25, 12489, Berlin, Germany. e-mail: ek@mathematik.hu-berlin.de

${ }^{\dagger}$ Department of Mathematical Sciences, Loughborough University, Loughborough, LE11 3TU, U.K. e-mail: a.b.pushnitski@lboro.ac.uk 
Thus, the determinant (1.2) is well defined.

(ii) The function $\Delta_{H / H_{0}}(z)$ is analytic in $z \in \mathbb{C}_{+}$and the limit $\Delta_{H / H_{0}}(\lambda+i 0)$ exists for all $\lambda \in \mathbb{R}$. (iii) The function $\Delta_{H / H_{0}}(\lambda+i 0)$ is locally Hölder continuous with any exponent $\gamma \in(0,1)$. I.e., for any $\gamma \in(0,1)$ and any $\lambda \in \mathbb{R}$, there exists a constant $C>0$ such that for all $\mu \in(\lambda-1, \lambda+1)$,

$$
\left|\Delta_{H / H_{0}}(\lambda+i 0)-\Delta_{H / H_{0}}(\mu+i 0)\right| \leq C|\lambda-\mu|^{\gamma} .
$$

(iv) One has

$$
\Delta_{H / H_{0}}(z) \neq 0 \quad \forall z \in \mathbb{C}_{+} \quad \text { and } \quad \Delta_{H / H_{0}}(\lambda+i 0) \neq 0, \quad \forall \lambda \in \mathbb{R} .
$$

The proof is a straightforward application of standard techniques of scattering theory. For completeness, we give the proof in the Appendix.

By (1.4), the analytic function $\log \Delta_{H / H_{0}}(z)$ (for some choice of the branch of the logarithm) is well defined. Changing the choice of the branch results in adding a constant to $\log \Delta_{H / H_{0}}(z)$. By Lemma 1.1(ii)-(iv), the limit $\log \Delta_{H / H_{0}}(\lambda+i 0)$ exists for all $\lambda \in \mathbb{R}$ and is a Hölder continuous function of $\lambda$. The derivative of $\log \Delta_{H / H_{0}}(z)$ admits a simple representation

$$
\frac{d}{d z} \log \Delta_{H / H_{0}}(z)=\operatorname{Tr}\left(\left(H_{0}-z\right)^{-1}-(H-z)^{-1}\right), \quad z \in \mathbb{C}_{+} ;
$$

this formula is valid for any pair of self-adjoint operators $H_{0}, H$ such that the inclusion (1.3) holds true.

4. The case $\boldsymbol{\beta}=0$. First we recall the large energy asymptotic expansion for $\log \Delta_{H / H_{0}}(z)$ in the case $\boldsymbol{\beta}=0$. Consider the operators $T_{0}=-\Delta$ and $T=-\Delta+V$ in $L^{2}\left(\mathbb{R}^{3}\right)$ (the potential $V$ is the same as in (1.1)) and denote

$$
\Delta_{T / T_{0}}(z)=\operatorname{det}\left(\frac{T-z}{T+i} \frac{T_{0}+i}{T_{0}-z}\right) .
$$

Similarly to Lemma 1.1, it is well known that the determinant $\Delta_{T / T_{0}}(z)$ is well defined, $\Delta_{T / T_{0}}(z) \neq$ 0 for $z \in \mathbb{C}_{+}$and $\Delta_{T / T_{0}}(z)$ is an analytic function of $z \in \mathbb{C}_{+}$. Let us define the function $\log \Delta_{T / T_{0}}(z)$ for some choice of the branch of the logarithm. We fix the branch of the square root of $z \in \mathbb{C}_{+}$by

$$
\sqrt{z}=\sqrt{|z|} e^{i \arg (z) / 2}, \quad \arg (z) \in[0, \pi] .
$$

It is well known that the following asymptotic expansion holds true for $\log \Delta_{T / T_{0}}(z)$ :

$$
\log \Delta_{T / T_{0}}(z) \sim i S_{-1} \sqrt{z}+C+\sum_{k=0}^{\infty} \frac{i S_{k}}{z^{k} \sqrt{z}}, \quad|z| \rightarrow \infty, \quad z \in \mathbb{C}_{+} .
$$

Here $C \in \mathbb{C}$ is a constant which depends on the choice of the branch of the logarithm in the definition of $\log \Delta_{T / T_{0}}(z)$. The coefficients $S_{k}$ are real numbers given by

$$
S_{k}=\int_{\mathbb{R}^{3}} F_{k}[V(x)] d x,
$$

and $F_{k}[V]$ are polynomials (with real coefficients) of $V$ and its derivatives. Explicit formulae for $F_{k}$ are known; in particular,

$$
F_{-1}[V]=\frac{1}{4 \pi} V, \quad F_{0}[V]=-\frac{1}{16 \pi} V^{2}, \quad F_{1}[V]=-\frac{1}{3 \cdot 2^{5} \pi} V^{3}-\frac{1}{3 \cdot 2^{6} \pi}|\nabla V|^{2} .
$$


The expansion (1.7) has been proven in $[5,6]$ (see also [7] and $[8,12,14]$ ). The expansion (1.7) can be considered as a consequence of the asymptotic expansion of the integral kernel of $(T-z)^{-1}$ on the diagonal (see (1.5)). A system of recurrence relations for computing the polynomials $F_{k}$ was given in [6]; explicit formulae can be found in the recent paper [10]. The coefficients $S_{k}$ appear also in the asymptotic expansion of $\operatorname{Tr}\left(e^{-t H}-e^{-t H_{0}}\right)$ as $t \rightarrow+0$; this connection gives an efficient way of computing $S_{k}$ (see e.g. [7]).

5. Asymptotic expansion for $\log \Delta_{H / H_{0}}(z)$. We return to the case $\boldsymbol{\beta} \neq 0$. Central to our construction is the asymptotic expansion of $\log \Delta_{H / H_{0}}(z)$ for $|z| \rightarrow \infty, z \in \mathbb{C}_{+}$. As above, we fix the branch of the square root by (1.6).

Theorem 1.2. The following asymptotic expansion holds true:

$$
\log \Delta_{H / H_{0}}(z) \sim i Q_{-1} \sqrt{z}+C+\sum_{k=0}^{\infty} \frac{i Q_{k}}{z^{k} \sqrt{z}}, \quad|z| \rightarrow \infty, \quad z \in \mathbb{C}_{+} .
$$

Here $C \in \mathbb{C}$ is a constant which depends on the choice of the branch of the logarithm in the definition of $\log \Delta_{H / H_{0}}(z)$. The coefficients $Q_{k}, k=-1,0,1,2, \ldots$ are real numbers given by

$$
Q_{k}=\int_{\mathbb{R}^{3}}\left(F_{k}[\langle\boldsymbol{\beta}, \mathbf{x}\rangle+V(\mathbf{x})]-F_{k}[\langle\boldsymbol{\beta}, \mathbf{x}\rangle]\right) d \mathbf{x},
$$

where $F_{k}$ are the polynomials from the expansion (1.7), (1.8).

In particular, by (1.9), one has

$$
\begin{aligned}
Q_{-1}= & \frac{1}{4 \pi} \int_{\mathbb{R}^{3}} V(\mathbf{x}) d \mathbf{x}, \\
Q_{0}= & -\frac{1}{16 \pi} \int_{\mathbb{R}^{3}} V^{2}(\mathbf{x}) d \mathbf{x}-\frac{1}{8 \pi} \int_{\mathbb{R}^{3}} V(\mathbf{x})\langle\boldsymbol{\beta}, \mathbf{x}\rangle d \mathbf{x}, \\
Q_{1}= & -\frac{1}{3 \cdot 2^{5} \pi} \int_{\mathbb{R}^{3}} V^{3}(\mathbf{x}) d \mathbf{x}-\frac{1}{3 \cdot 2^{6} \pi} \int_{\mathbb{R}^{3}}|\nabla V(\mathbf{x})|^{2} d \mathbf{x}-\frac{1}{2^{5} \pi} \int_{\mathbb{R}^{3}} V^{2}(\mathbf{x})\langle\boldsymbol{\beta}, \mathbf{x}\rangle d \mathbf{x} \\
& -\frac{1}{2^{5} \pi} \int_{\mathbb{R}^{3}} V(\mathbf{x})\langle\boldsymbol{\beta}, \mathbf{x}\rangle^{2} d \mathbf{x} .
\end{aligned}
$$

The important feature of the expansion (1.10) is that it holds uniformly over $\arg (z) \in(0, \pi)$, and thus also holds for the boundary values $\log \Delta_{H / H_{0}}(\lambda+i 0)$.

The coefficient $C$ in (1.10) is not important for our construction; essentially, we are working with the function

$$
F(z):=\log \Delta_{H / H_{0}}(z)-C .
$$

We use the following method of proof of the expansion (1.10), (1.11). In Section 3, using a method similar to the one of [7], we prove that the asymptotic expansion (1.10) holds true with some coefficients $Q_{k} \in \mathbb{C}$; the approach of Section 3 does not give simple formulae (1.11) for these coefficients. Next, in Section 4, using different technique, we prove that in any sector $\{z \in \mathbb{C} \mid \varepsilon<\arg z<\pi-\varepsilon\}, \varepsilon>0$, the expansion (1.10) holds true with the coefficients $Q_{k}$ given by (1.11); the approach of Section 4 does not allow us to prove the expansion (1.10) uniformly over $\arg (z) \in(0, \pi)$. By uniqueness of the coefficients of an asymptotic expansion, the combination of the two above statements leads to Theorem 1.2. 
Note that the operators $H_{0}$ and $H$ are not semi-bounded from below, which makes it difficult, if not impossible, to use the corresponding heat kernels $e^{-t H}, e^{-t H_{0}}$ for computing the coefficients $Q_{k}$ (as it can be done for the case $\boldsymbol{\beta}=0$ ). In this paper, we work with resolvents rather than with heat kernels.

6. The spectral shift function. It appears that $\log \Delta_{H / H_{0}}(z)$ admits the integral representation

$$
\log \Delta_{H / H_{0}}(z)=\int_{-\infty}^{\infty} \xi(\lambda)\left(\frac{1}{\lambda-z}-\frac{1}{\lambda+i}\right) d \lambda
$$

where $\xi$ is the spectral shift function for the pair (1.1). We refer to the survey paper [3] or to the book [16] for exposition of the spectral shift function theory. The spectral shift function is related to the scattering matrix of the pair (1.1); we postpone the discussion of this subject till Section 5 .

By (1.13), the spectral shift function can be determined by the formula

$$
\xi(\lambda)=\frac{1}{\pi} \operatorname{Im} \log \Delta_{H / H_{0}}(\lambda+i 0)-\frac{1}{2 \pi} \operatorname{Im} \log \Delta_{H / H_{0}}(i), \quad \lambda \in \mathbb{R} .
$$

The change of the branch of the logarithm results in adding an integer constant to $\xi$. In order to fix this constant, we need the following

Lemma 1.3. One has

$$
\xi(\lambda)=n+O\left(|\lambda|^{-N}\right), \quad \lambda \rightarrow-\infty, \quad \forall N>0,
$$

where $n$ is an integer, which depends on the choice of the branch of the logarithm in (1.13).

The proof is given in Section 5 .

Clearly, we can choose the branch of the logarithm so that the constant $n$ in (1.15) equals zero. Thus, $\xi$ is normalized by

$$
\xi(\lambda) \rightarrow 0, \quad \lambda \rightarrow-\infty .
$$

In terms of the function $F(z)$ (see (1.12)) this implies

$$
\xi(\lambda)=\frac{1}{\pi} \operatorname{Im} F(\lambda+i 0), \quad \lambda \in \mathbb{R} .
$$

The asymptotic expansion of Theorem 1.2 immediately yields the large energy asymptotic expansion for $\xi$ :

$$
\begin{aligned}
& \xi(\lambda) \sim \frac{1}{\pi} \sum_{k=-1}^{\infty} \frac{Q_{k}}{\lambda^{k} \sqrt{\lambda}}, \quad \lambda \rightarrow+\infty \\
& \xi(\lambda)=O\left(|\lambda|^{-N}\right), \quad \lambda \rightarrow-\infty, \quad \forall N>0 .
\end{aligned}
$$

The first term in the expansion (1.18) has been obtained earlier in [15] for a much wider class of potentials $V$. It has been also proven in [15] that $\xi(\lambda) \rightarrow 0$ as $\lambda \rightarrow-\infty$. Note that the paper [15] deals with the case of an arbitrary dimension.

7. The harmonic conjugate $h$. Apart from $\xi(\lambda)$, the trace formulae for the pair (1.1) involve a function $h(\lambda)$, which is a properly normalized harmonic conjugate of $\xi(\lambda)$. We define $h(\lambda)$ by (compare with (1.17))

$$
h(\lambda)=\frac{1}{\pi} \operatorname{Re} F(\lambda+i 0), \quad \lambda \in \mathbb{R} .
$$


By Lemma 1.1(iii),(iv), the function $h(\lambda)$ is Hölder continuous. The asymptotic expansion of Theorem 1.2 yields (compare with (1.18),(1.19))

$$
\begin{aligned}
& h(\lambda)=O\left(|\lambda|^{-N}\right), \quad \lambda \rightarrow+\infty, \quad \forall N>0, \\
& h(\lambda) \sim \frac{1}{\pi} \sum_{k=-1}^{\infty} \frac{Q_{k}}{\lambda^{k} \sqrt{|\lambda|}}, \quad \lambda \rightarrow-\infty .
\end{aligned}
$$

Remark One can define the spectral shift function by using the Birman-Krein formula (see the discussion in Section 5) without any reference to the perturbation determinant. Then $h(\lambda)$ can also be defined without any reference to $\Delta_{H / H_{0}}(z)$ by the following two conditions:

(i) $h(\lambda)+i \xi(\lambda)$ is a boundary value of an analytic function in $\mathbb{C}_{+}$;

(ii) the following normalization condition holds true: $h(\lambda) \rightarrow 0$ as $\lambda \rightarrow+\infty$.

8. Trace formulae. Now that $\xi$ and $h$ have been defined, we are ready to state the result on the trace formulae. Let $Q_{k}$ be the coefficients from the expansion (1.10).

Theorem 1.4. The following trace formulae hold true:

$$
\begin{gathered}
Q_{-1}=\int_{0}^{\infty} \frac{h(\lambda)-h(0)}{\lambda \sqrt{\lambda}} d \lambda-\int_{-\infty}^{0} \frac{\xi(\lambda)-\xi(0)}{|\lambda| \sqrt{|\lambda|}} d \lambda \\
Q_{n}=\int_{0}^{\infty} h(\lambda) \lambda^{n-\frac{1}{2}} d \lambda+(-1)^{n} \int_{-\infty}^{0}|\lambda|^{n-\frac{1}{2}} \xi(\lambda) d \lambda, \quad n=0,1,2, \ldots \\
\int_{-\infty}^{\infty}\left(\xi(\lambda)-\frac{1}{\pi} \sum_{k=-1}^{n} \frac{Q_{k}}{\lambda^{k} \sqrt{\lambda}} \theta(\lambda)\right) \lambda^{n} d \lambda=0, \quad n=1,2, \ldots
\end{gathered}
$$

Note that by (1.18), (1.19) and (1.20), the above integrals converge absolutely.

Given the asymptotic expansion (1.10), the proof of the trace formulae (1.22)-(1.24) becomes a simple exercise in complex analysis (see e.g. [6, 7]). For completeness, we give this proof in Section 2.

Trace formulae for the case $\boldsymbol{\beta}=0$ were obtained by V. S. Buslaev and L. D. Faddeev in [4] in the one-dimensional situation and by V. S. Buslaev in $[5,6]$ in the three-dimensional situation. In the case $\boldsymbol{\beta}=0$ the integrals over $(-\infty, 0)$ can be replaced by the sums of the appropriate powers of eigenvalues of $-\Delta+V$.

9. Remark Fix any $a \in \mathbb{R}$. The asymptotic expansion (1.10) is equivalent to the asymptotic expansion in powers of $(z-a)$ :

$$
\log \Delta_{H / H_{0}}(z) \sim i Q_{-1}^{(a)} \sqrt{z-a}+C+\sum_{k=0}^{\infty} \frac{i Q_{k}^{(a)}}{(z-a)^{k} \sqrt{z-a}}, \quad|z| \rightarrow \infty, \quad z \in \mathbb{C}_{+} .
$$

The coefficients $Q_{k}^{(a)}$ are real and can be expressed in terms of the coefficients $Q_{k}$. For example,

$$
Q_{-1}^{(a)}=Q_{-1}, \quad Q_{0}^{(a)}=Q_{0}+\frac{a}{2} Q_{-1}, \quad Q_{1}^{(a)}=Q_{1}-\frac{a}{2} Q_{0}-\frac{a^{2}}{8} Q_{-1} .
$$

In the same way as the expansion (1.10) yields the trace formulae (1.22)-(1.24), the expansion 
(1.25) yields

$$
\begin{gathered}
Q_{-1}=\int_{a}^{\infty} \frac{h(\lambda)-h(a)}{(\lambda-a) \sqrt{\lambda-a}} d \lambda-\int_{-\infty}^{a} \frac{\xi(\lambda)-\xi(a)}{|\lambda-a| \sqrt{|\lambda-a|}} d \lambda, \\
Q_{n}^{(a)}=\int_{a}^{\infty} h(\lambda)(\lambda-a)^{n-\frac{1}{2}} d \lambda+(-1)^{n} \int_{-\infty}^{a}|\lambda-a|^{n-\frac{1}{2}} \xi(\lambda) d \lambda, \quad n=0,1,2, \ldots \\
\int_{-\infty}^{\infty}\left(\xi(\lambda)-\frac{1}{\pi} \sum_{k=-1}^{n} \frac{Q_{k}^{(a)}}{(\lambda-a)^{k} \sqrt{\lambda-a}} \theta(\lambda-a)\right)(\lambda-a)^{n} d \lambda=0, \quad n=1,2, \ldots
\end{gathered}
$$

10. The structure of the paper. In Section 2 we prove Theorem 1.4, assuming Theorem 1.2 and Lemma 1.3. In Sections 3 and 4 we prove Theorem 1.2. In Section 5 we recall the connection between the spectral shift function and the scattering matrix, and, using this connection, prove Lemma 1.3. In Appendix we prove Lemma 1.1.

\section{Proof of Theorem 1.4}

1. Proof of (1.22). Let $F$ be the function defined by (1.12). Then $\frac{1}{\pi} F(\lambda+i 0)=h(\lambda)+i \xi(\lambda)$ for all $\lambda \in \mathbb{R}$. By Lemma 1.1(iii),(iv), the function $F(\lambda+i 0)$ is Hölder continuous.

Next, consider an auxiliary function

$$
L(z)=i \frac{F(z)-F(0)}{z \sqrt{z}} .
$$

The function $L$ is analytic in $\mathbb{C}_{+}$; the asymptotic expansion of Theorem 1.2 yields

$$
L(z)=-\frac{Q_{-1}}{z}+O\left(|z|^{-3 / 2}\right), \quad|z| \rightarrow \infty, \quad z \in \mathbb{C}_{+} .
$$

The boundary values $L(\lambda+i 0)$ belong to $L^{p}(\mathbb{R})$ for any $p \in(1,2)$ (by the Hölder continuity of $F)$. Thus, $L$ belongs to the Hardy class $H^{p}\left(\mathbb{C}_{+}\right), p \in(1,2)$ and therefore can be represented as a Cauchy type integral

$$
L(z)=\frac{1}{\pi} \int_{-\infty}^{\infty} \frac{\operatorname{Im} L(\lambda+i 0)}{\lambda-z} d \lambda
$$

From here we get

$$
L(z)=-\frac{1}{\pi z} \int_{-\infty}^{\infty} \operatorname{Im} L(\lambda+i 0) d \lambda+o\left(|z|^{-1}\right), \quad \operatorname{Im} z \rightarrow+\infty, \quad z \in \mathbb{C}_{+} .
$$

Comparing this to (2.1), one obtains

$$
\begin{aligned}
Q_{-1}=\frac{1}{\pi} \int_{-\infty}^{\infty} \operatorname{Im} L(\lambda+i 0) d \lambda=\int_{-\infty}^{\infty} \operatorname{Im}\left(i \frac{h(\lambda)+i \xi(\lambda)-h(0)-i \xi(0)}{\lambda \sqrt{\lambda}}\right) d \lambda & \\
& =\int_{0}^{\infty} \frac{h(\lambda)-h(0)}{\lambda \sqrt{\lambda}} d \lambda-\int_{-\infty}^{0} \frac{\xi(\lambda)-\xi(0)}{|\lambda| \sqrt{|\lambda|}} d \lambda .
\end{aligned}
$$

2. Proof of (1.23). Consider an auxiliary function

$$
L(z)=i z^{n-1} \sqrt{z}\left(F(z)-\sum_{k=-1}^{n-1} \frac{i Q_{k}}{z^{k} \sqrt{z}}\right) .
$$


The function $L$ is analytic in $\mathbb{C}_{+}$; the asymptotic expansion of Theorem 1.2 yields

$$
L(z)=-\frac{Q_{n}}{z}+O\left(|z|^{-2}\right), \quad|z| \rightarrow \infty, \quad z \in \mathbb{C}_{+} .
$$

The boundary values $L(\lambda+i 0)$ belong to $L^{p}(\mathbb{R})$ for any $p>1$ if $n>0$ and for any $p \in(1,2)$ if $n=0$. Thus, $L$ belongs to the Hardy class $H^{p}\left(\mathbb{C}_{+}\right)$, the integral representation $(2.2)$ holds true and we get (2.3). Comparing (2.3) and (2.4), one obtains

$Q_{n}=\frac{1}{\pi} \int_{-\infty}^{\infty} \operatorname{Im}\left[i z^{n-1} \sqrt{z}\left(F(z)-\sum_{k=-1}^{n-1} \frac{i Q_{k}}{z^{k} \sqrt{z}}\right)\right]=\int_{0}^{\infty} h(\lambda) \lambda^{n-\frac{1}{2}} d \lambda+(-1)^{n} \int_{-\infty}^{0} \xi(\lambda)|\lambda|^{n-\frac{1}{2}} d \lambda$.

3. Proof of (1.24). Consider an auxiliary function

$$
L(z)=z^{n}\left(F(z)-\sum_{k=-1}^{n} \frac{i Q_{k}}{z^{k} \sqrt{z}}\right) .
$$

The function $L$ is analytic in $\mathbb{C}_{+}$; the asymptotic expansion of Theorem 1.2 yields

$$
L(z)=O\left(|z|^{-3 / 2}\right), \quad|z| \rightarrow \infty, \quad z \in \mathbb{C}_{+} .
$$

The function $L$ belongs to the Hardy class $H^{p}\left(\mathbb{C}_{+}\right)$for any $p \in[1,2)$. As above, the integral representation (2.2) and the asymptotic formula (2.3) hold true; comparing (2.3) and (2.5), we get

$$
0=\frac{1}{\pi} \int_{-\infty}^{\infty} \operatorname{Im}\left(\lambda^{n} F(\lambda)-\lambda^{n} \sum_{k=-1}^{n} \frac{i Q_{k}}{\lambda^{k} \sqrt{\lambda}}\right) d \lambda=\int_{-\infty}^{\infty}\left(\xi(\lambda)-\frac{1}{\pi} \sum_{k=-1}^{n} \frac{Q_{k}}{\lambda^{k} \sqrt{\lambda}} \theta(\lambda)\right) \lambda^{n} d \lambda .
$$

This completes the proof.

\section{Proof of the expansion (1.10)}

In this section we prove that the expansion (1.10) holds true with some coefficients $Q_{k} \in \mathbb{C}$. Formulae (1.11) for these coefficients will be proven in the next section.

1. Notation Throughout the rest of the paper we use the following notation. For the vector $\boldsymbol{\beta}$ from the definition of $H_{0}$, we denote $\beta=|\boldsymbol{\beta}|$. For a vector $\mathbf{x} \in \mathbb{R}^{3}$, we denote by $x^{\|} \in \mathbb{R}$ and $x^{\perp} \in \mathbb{R}^{2}$ the components of $\mathbf{x}$ parallel and orthogonal to the direction of $\boldsymbol{\beta}$.

We factorize the potential $V$ as follows:

$$
V=W_{1} W_{2}, \quad W_{1}=\sqrt{|V|}, \quad W_{2}=\operatorname{sign}(V) \sqrt{|V|} .
$$

Our main objects in this section are the free resolvent $R_{0}(z)=\left(H_{0}-z\right)^{-1}$ and the sandwiched resolvent $T(z)=W_{1} R_{0}(z) W_{2}$.

2. Strategy Our reasoning is similar to that of [7] and is based on the representation

$$
\log \Delta_{H / H_{0}}(z)=\log \Delta_{H / H_{0}}(i)+\int_{i}^{z} \operatorname{Tr}\left(\frac{d}{d w} T(w)\right) d w+\sum_{n=1}^{\infty} \frac{(-1)^{n}}{n+1} \operatorname{Tr} T^{n+1}(z),
$$


which holds true for all $z \in \mathbb{C}_{+}$far enough from the origin. The representation (3.2) is essentially well known (see e.g. [7]), but for completeness we give a proof below. In order to derive (1.10) from (3.2), we will need (i) estimates for the remainder of the series in the r.h.s. of (3.2); (ii) asymptotic expansion for large $|z|$ of every term in the r.h.s. of (3.2).

3. Estimates for $T(z)$ We will need estimates of the operator norm $\|T(z)\|$ and the HilbertSchmidt norm $\|T(z)\|_{\mathfrak{S}_{2}}$ of $T(z)$.

Lemma 3.1. [11] For any $\varepsilon \in(0,1 / 4)$,

$$
\|T(z)\| \leq C(1+|z|)^{-\frac{1}{4}+\varepsilon}, \quad z \in \mathbb{C}_{+},
$$

where the constant $C$ depends on $V, \beta$, and $\varepsilon$, but not on $z$.

Lemma 3.2. For any $z \in \mathbb{C}_{+}$, the sandwiched resolvent $T(z)$ belongs to the Hilbert-Schmidt class and

$$
\|T(z)\|_{\mathfrak{S}_{2}}^{2} \leq C_{1} \int_{\mathbb{R}^{3}} \int_{\mathbb{R}^{3}} \frac{|V(\mathbf{x}) V(\mathbf{y})|}{|\mathbf{x}-\mathbf{y}|^{2}} d \mathbf{x} d \mathbf{y}+C_{2} \beta^{2 / 3}\left(\int_{\mathbb{R}^{3}}|V(x)| d \mathbf{x}\right)^{2}
$$

where $C_{1}$ and $C_{2}$ are absolute constants.

(The actual expression in the r.h.s. of (3.3) is of no importance to our construction; it is essential that this expression does not depend on $z$ ).

Proof. For the resolvent $R_{0}(z)$ one has

$$
R_{0}(z)=i \int_{0}^{\infty} e^{i z t} e^{-i t H_{0}} d t
$$

Using the Avron-Herbst formula for $e^{-i t H_{0}}$ (see [1]), one finds the integral kernel $R_{0}(z)[\mathbf{x}, \mathbf{y}]$ of $R_{0}(z)$ :

$$
R_{0}(z)[\mathbf{x}, \mathbf{y}]=\frac{e^{-i \pi / 4}}{8 \pi \sqrt{\pi}} \int_{0}^{\infty} t^{-3 / 2} e^{-i \beta^{2} t^{3} / 12} e^{i z t} e^{-i\left(x^{\|}+y^{\|}\right) \beta t / 2} e^{i|\mathbf{x}-\mathbf{y}|^{2} / 4 t} d t .
$$

By the same pattern, for any $\eta \in \mathbb{C}_{+}$one has the following representation for the integral kernel of the resolvent $(-\Delta-\eta)^{-1}$ in $L^{2}\left(\mathbb{R}^{3}\right)$ :

$$
\frac{e^{i \sqrt{\eta}|\mathbf{x}-\mathbf{y}|}}{4 \pi|\mathbf{x}-\mathbf{y}|}=\frac{e^{-i \pi / 4}}{8 \pi \sqrt{\pi}} \int_{0}^{\infty} t^{-3 / 2} e^{i \eta t} e^{i|\mathbf{x}-\mathbf{y}|^{2} / 4 t} d t .
$$

Denoting $\eta:=z-\left(x^{\|}+y^{\|}\right) \beta / 2$ and subtracting (3.5) from (3.4), we obtain:

$$
R_{0}(z)[\mathbf{x}, \mathbf{y}]=\frac{e^{i \sqrt{\eta}|\mathbf{x}-\mathbf{y}|}}{4 \pi|\mathbf{x}-\mathbf{y}|}+\frac{e^{-i \pi / 4}}{8 \pi \sqrt{\pi}} \int_{0}^{\infty} t^{-3 / 2}\left(e^{-i \beta^{2} t^{3} / 12}-1\right) e^{i \eta t} e^{i|\mathbf{x}-\mathbf{y}|^{2} / 4 t} d t
$$

Thus,

$$
\begin{aligned}
\left|R_{0}(z)[\mathbf{x}, \mathbf{y}]\right| & \leq \frac{1}{4 \pi|\mathbf{x}-\mathbf{y}|}+\frac{1}{8 \pi \sqrt{\pi}} \int_{0}^{\infty} t^{-3 / 2}\left|e^{-i \beta^{2} t^{3} / 12}-1\right| d t \\
& =\frac{1}{4 \pi|\mathbf{x}-\mathbf{y}|}+\beta^{1 / 3} \frac{1}{8 \pi \sqrt{\pi}} \int_{0}^{\infty} t^{-3 / 2}\left|e^{-i t^{3} / 12}-1\right| d t
\end{aligned}
$$

Now a direct estimate of the Hilbert-Schmidt norm of $T(z)$ yields (3.3). 
4. Asymptotics for $T(z)$. Below we use the notation $T^{\prime}(z):=\frac{d}{d z} T(z)=W_{1} R_{0}^{2}(z) W_{2}$. It is easy to show (see, e.g., the proof of Theorem 4.2 in [1]) that

$$
W_{1}\left(H_{0}-z\right)^{-1} \in \mathfrak{S}_{2}, \quad \forall z \in \mathbb{C}_{+} .
$$

From here it follows that the operator $T^{\prime}(z)$ belongs to the trace class.

Lemma 3.3. The following asymptotic expansion holds true:

$$
\operatorname{Tr} T^{\prime}(z) \sim \sum_{k=0}^{\infty} \frac{i a_{k}}{z^{k} \sqrt{z}}, \quad|z| \rightarrow \infty, \quad z \in \mathbb{C}_{+},
$$

with some coefficients $a_{k} \in \mathbb{C}$.

Lemma 3.4. For any $n=2,3, \ldots$, the following asymptotic expansion holds true:

$$
\operatorname{Tr}(T(z))^{n} \sim \sum_{k=n-2}^{\infty} \frac{i a_{k}^{(n)}}{z^{k} \sqrt{z}}, \quad|z| \rightarrow \infty, \quad z \in \mathbb{C}_{+},
$$

with some coefficients $a_{k}^{(n)} \in \mathbb{C}$.

We postpone the proof of Lemmas 3.3 and 3.4 till the end of this section.

5. Proof of the expansion (1.10) First note that a simple computation (see formula (6.1) in the Appendix) yields

$$
\Delta_{H / H_{0}}(z)=\operatorname{det}(I+T(z))(I+T(-i))^{-1} .
$$

Next, by Lemma 3.1, one can choose $R>0$ big enough so that

$$
\|T(z)\| \leq 1 / 2 \quad \forall z \in \mathbb{C}_{+}, \quad|z|>R .
$$

For such $z$, differentiating $\log \Delta_{H / H_{0}}(z)$ and expanding the result, we get

$$
\begin{aligned}
\frac{d}{d z} \log \Delta_{H / H_{0}}(z)=\frac{d}{d z} \log \operatorname{det}(I+ & T(z))(I+T(-i))^{-1} \\
& =\operatorname{Tr}\left(T^{\prime}(z)(I+T(z))^{-1}\right)=\sum_{n=0}^{\infty}(-1)^{n} \operatorname{Tr}\left(T^{\prime}(z) T^{n}(z)\right),
\end{aligned}
$$

where the series converge absolutely by (3.9):

$$
\left|\operatorname{Tr} T^{\prime}(z) T^{n}(z)\right| \leq\left\|T^{\prime}(z)\right\|_{\mathfrak{S}_{1}} \cdot 2^{-n} .
$$

Integrating (3.10), one obtains (3.2). Note that by Lemma 3.1, Lemma 3.2 and (3.9), one has the following bound on the remainder of the series in the r.h.s. of (3.2):

$$
\begin{aligned}
&\left|\sum_{n=N+1}^{\infty} \frac{(-1)^{n}}{n+1} \operatorname{Tr} T^{n+1}(z)\right| \leq\|T(z)\|_{\mathfrak{S}_{2}}^{2} \sum_{n=N+1}^{\infty}\|T(z)\|^{n-1} \leq 2\|T(z)\|_{\mathfrak{S}_{2}}^{2}\|T(z)\|^{N} \\
&=O\left(|z|^{-\left(\frac{1}{4}-\varepsilon\right) N}\right), \quad \forall \varepsilon \in(0,1 / 4), \quad N=0,1,2, \ldots
\end{aligned}
$$


Due to the last estimate, the required asymptotic expansion (1.10) follows from the convergent series expansion (3.2) and the asymptotic expansions given by Lemma 3.3 and Lemma 3.4.

\section{Proof of Lemma 3.3.}

1. Integral representation for $\operatorname{Tr} T^{\prime}(z)$ Differentiating (3.4) over $z$, we obtain the formula for the integral kernel of $R_{0}^{2}(z)$ :

$$
R_{0}^{2}(z)[\mathbf{x}, \mathbf{y}]=\frac{e^{i \pi / 4}}{8 \pi \sqrt{\pi}} \int_{0}^{\infty} t^{-1 / 2} e^{-i \beta^{2} t^{3} / 12} e^{i z t} e^{-i\left(x^{\|}+y^{\|}\right) \beta t / 2} e^{i|\mathbf{x}-\mathbf{y}|^{2} / 4 t} d t .
$$

Now $\operatorname{Tr} T^{\prime}(z)$ can be explicitly computed:

$$
\begin{aligned}
\operatorname{Tr} T^{\prime}(z) & =\int_{\mathbb{R}^{3}} V(\mathbf{x}) R_{0}^{2}(z)[\mathbf{x}, \mathbf{x}] d \mathbf{x}=\frac{e^{i \pi / 4}}{8 \pi \sqrt{\pi}} \int_{\mathbb{R}^{3}} d \mathbf{x} V(\mathbf{x}) \int_{0}^{\infty} d t t^{-1 / 2} e^{-i \beta^{2} t^{3} / 12} e^{i z t} e^{-i x \|^{\|}} \\
& =\frac{e^{i \pi / 4}}{2 \sqrt{2}} \int_{0}^{\infty} \widehat{V}(t \boldsymbol{\beta}) e^{-i \beta^{2} t^{3} / 12} e^{i z t} t^{-1 / 2} d t
\end{aligned}
$$

where $\widehat{V}$ is the (unitary) Fourier transform of $V$.

2. Stationary phase expansion Denote $g(t)=\dot{\widehat{V}}(t \boldsymbol{\beta}) e^{-i \beta^{2} t^{3} / 12}$. Due to our assumption $V \in$

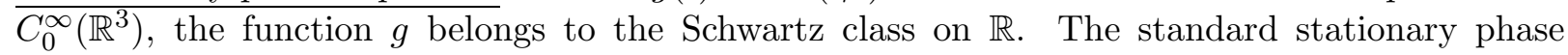
technique gives the expansion

$$
\int_{0}^{\infty} g(t) e^{i z t} t^{-1 / 2} d t \sim e^{i \pi / 4} \sum_{k=0}^{\infty} \frac{i^{k} \Gamma\left(k+\frac{1}{2}\right)}{k ! z^{k} \sqrt{z}} g^{(k)}(0), \quad|z| \rightarrow \infty, \quad z \in \mathbb{C}_{+} .
$$

By (3.11), this yields the required expansion (3.7).

\section{Proof of Lemma 3.4}

1. Strategy The strategy of the proof is the same as that of the proof of Lemma 3.3, but the technical details are more complicated. We are going to prove the following integral representation:

$$
\operatorname{Tr} T^{n}(z)=\int_{0}^{\infty} t^{-1 / 2} e^{i z t} g(t) d t, \quad g(t)=t^{n-2} f(t),
$$

where $f$ is a Schwartz class function on $\mathbb{R}$. The stationary phase method gives the expansion (3.12). As $g^{(k)}(0)=0$ for $k=0,1, \ldots, n-3$, the expansion actually starts from the term with $k=n-2$, so we get (3.8).

2. The kernel of $\Phi R_{0}(z) V \Phi^{*}$ First note that by $(3.6),\left(R_{0}(z) V\right)^{n}$ is a trace class operator for $n \geq 2$, and so $\operatorname{Tr} T^{n}(z)=\operatorname{Tr}\left(R_{0}(z) V\right)^{n}$. Next, $\operatorname{Tr}\left(R_{0}(z) V\right)^{n}=\operatorname{Tr}\left(\Phi R_{0}(z) V \Phi^{*}\right)^{n}$, where $\Phi$ is the (unitary) Fourier transform in $L^{2}\left(\mathbb{R}^{3}\right)$. Let us compute the integral kernel of the operator $\Phi R_{0}(z) V \Phi^{*}$. For the resolvent $R_{0}(z)$ one has (see [1]):

$$
R_{0}(z)=\Phi^{*} e^{i\left(x^{\|}\right)^{3} / 3 \beta}\left(i \beta \frac{\partial}{\partial x^{\|}}+\left|x^{\perp}\right|^{2}-z\right)^{-1} e^{-i\left(x^{\|}\right)^{3} / 3 \beta} \Phi .
$$

The integral kernel of the resolvent of $i \beta \frac{\partial}{\partial x \|}$ can be easily computed. This gives the following expression for the integral kernel of $\Phi R_{0}(z) V \Phi^{*}$ :

$$
\begin{gathered}
\left(\Phi R_{0}(z) V \Phi^{*}\right)[\mathbf{x}, \mathbf{y}]=i(2 \pi)^{-3 / 2} \int_{0}^{\infty} \widehat{V}(\mathbf{x}-\mathbf{y}+\beta t) e^{i z t} e^{-i \phi_{0}}, \\
\phi_{0}=\frac{1}{3 \beta}\left(x^{\|}-\beta t\right)^{3}-\frac{1}{3 \beta}\left(x^{\|}\right)^{3}+t\left|x^{\perp}\right|^{2}=t|\mathbf{x}|^{2}+\beta t^{2} x^{\|}+\beta^{2} t^{3} / 3 .
\end{gathered}
$$


Iterating the above kernel and computing trace, one obtains:

$$
\begin{aligned}
\operatorname{Tr}\left(\Phi R_{0}(z) V \Phi^{*}\right)^{n}= & i^{n}(2 \pi)^{-3 n / 2} \int_{\mathbb{R}^{3}} d \mathbf{x}_{1}\left[\int_{0}^{\infty} d t_{1} \ldots \int_{0}^{\infty} d t_{n} \int_{\mathbb{R}^{3}} d \mathbf{x}_{2} \ldots \int_{\mathbb{R}^{3}} d \mathbf{x}_{n}\right. \\
\times & \left.e^{i z\left(t_{1}+\cdots+t_{n}\right)} \widehat{V}\left(\mathbf{x}_{1}-\mathbf{x}_{2}+t_{1} \boldsymbol{\beta}\right) \ldots \widehat{V}\left(\mathbf{x}_{n}-\mathbf{x}_{1}+t_{n} \boldsymbol{\beta}\right) e^{-i \phi}\right], \\
\phi & =\sum_{j=1}^{n} t_{j}\left|\mathbf{x}_{j}\right|^{2}+\beta \sum_{j=1}^{n} t_{j}^{2} x_{j}^{\|}+\left(\beta^{2} / 3\right) \sum_{j=1}^{n} t_{j}^{3} .
\end{aligned}
$$

It is easy to see that the integral in the square brackets in (3.16) converges absolutely. The integral over $\mathbf{x}_{1}$ also converges absolutely (as $\left(\Phi R_{0}(z) V \Phi^{*}\right)^{n}$ belongs to the trace class), but we may not be able to legitimately interchange the order of integration. As we still would like to do this, we use the following regularization:

$$
\operatorname{Tr}\left(\Phi R_{0}(z) V \Phi^{*}\right)^{n}=i^{n}(2 \pi)^{-3 n / 2} \lim _{R \rightarrow \infty} \int_{[-R, R]^{3}} d \mathbf{x}_{1}[\cdots]
$$

where $[\ldots]$ stands for the integral in the square brackets in (3.16).

3. Change of variables Let us perform the following changes of variables in the integral (3.16). First, instead of $t_{1}, \ldots, t_{n}$, we introduce the variables $t_{0}, \omega_{1}, \omega_{2}, \ldots, \omega_{n-1}$,

$$
t_{0}=t_{1}+\cdots+t_{n}, \quad \omega_{j}=t_{j} / t_{0}, \quad j=1,2, \ldots, n-1 .
$$

We also use the notation $\omega_{n}:=1-\omega_{1}-\cdots-\omega_{n-1}$, but $\omega_{n}$ is treated as a function of the variables $\omega_{1}, \omega_{2}, \ldots, \omega_{n-1}$, rather than as an independent variable. We denote by $\omega$ the vector $\omega=\left(\omega_{1}, \ldots, \omega_{n-1}\right) \in \mathbb{R}^{n-1}$. The integral over $d t_{1} \ldots d t_{n}$ then becomes

$$
\int_{0}^{\infty} d t_{1} \ldots \int_{0}^{\infty} d t_{n}=\int_{0}^{\infty} d t_{0} t_{0}^{n-1} \int_{0}^{1} d \omega_{1} \int_{0}^{1-\omega_{1}} d \omega_{2} \int_{0}^{1-\omega_{1}-\omega_{2}} d \omega_{3} \ldots \int_{0}^{1-\omega_{1}-\cdots-\omega_{n-2}} d \omega_{n-1}
$$

we will use the shorthand notation $\int_{\Omega} d \omega$ for the above integral over $d \omega_{1} \ldots d \omega_{n-1}$.

Next, instead of $\mathbf{x}_{1}, \ldots, \mathbf{x}_{n}$, we introduce the variables

$$
\mathbf{x}_{0}=\mathbf{x}_{1}+\cdots+\mathbf{x}_{n}, \quad \mathbf{y}_{j}=\mathbf{x}_{j}-\mathbf{x}_{j+1}+t_{0} \omega_{j} \boldsymbol{\beta}, \quad j=1, \ldots, n-1 .
$$

We also use the notation $y^{\|}=\left(y_{1}^{\|}, y_{2}^{\|}, \ldots, y_{n-1}^{\|}\right) \in \mathbb{R}^{n-1}, y^{\perp}=\left(y_{1}^{\perp}, y_{2}^{\perp}, \ldots, y_{n-1}^{\perp}\right) \in \mathbb{R}^{2(n-1)}$, $y=\left(\mathbf{y}_{1}, \mathbf{y}_{2} \ldots, \mathbf{y}_{n-1}\right) \in \mathbb{R}^{3(n-1)}$.

Expressing $\mathbf{x}_{1}, \ldots, \mathbf{x}_{n}$ in terms of the new variables, we get

$$
\begin{aligned}
x_{j}^{\|} & =\frac{1}{n} x_{0}^{\|}+l_{j}^{\|}\left(y^{\|}-\beta t_{0} \omega\right), \quad j=1, \ldots, n, \\
x_{j}^{\perp} & =\frac{1}{n} x_{0}^{\perp}+l_{j}^{\perp}\left(y^{\perp}\right), \quad j=1, \ldots, n,
\end{aligned}
$$

where $l_{j}^{\|}: \mathbb{R}^{n-1} \rightarrow \mathbb{R}$ and $l_{j}^{\perp}: \mathbb{R}^{2(n-1)} \rightarrow \mathbb{R}^{2}$ are some linear functions. The integral over $d \mathbf{x}_{1} \ldots d \mathbf{x}_{n}$ can be transformed as

$$
\int_{[-R, R]^{3}} d \mathbf{x}_{1} \int_{\mathbb{R}^{3}} d \mathbf{x}_{2} \cdots \int_{\mathbb{R}^{3}} d \mathbf{x}_{n}=\frac{1}{n^{3}} \int_{\mathbb{R}^{3}} d \mathbf{y}_{1} \cdots \int_{\mathbb{R}^{3}} d \mathbf{y}_{n-1} \int_{Q_{R}} d \mathbf{x}_{0},
$$


where $Q_{R}=[-n R, n R]^{3}-\left(n l_{1}^{\|}\left(y^{\|}-\beta t_{0} \omega\right), n l_{1}^{\perp}\left(y^{\perp}\right)\right)$. Thus, we get

$$
\begin{aligned}
\operatorname{Tr} & \left(\Phi R_{0}(z) V \Phi^{*}\right)^{n}=i^{n}(2 \pi)^{-3 n / 2} n^{-3} \lim _{R \rightarrow \infty} \int_{0}^{\infty} d t_{0} t_{0}^{n-1} e^{i z t_{0}} \int_{\Omega} d \omega \\
& \times \int_{\mathbb{R}^{3}} d \mathbf{y}_{1} \ldots \int_{\mathbb{R}^{3}} d \mathbf{y}_{n-1} \widehat{V}\left(\mathbf{y}_{1}\right) \ldots \widehat{V}\left(\mathbf{y}_{n-1}\right) \widehat{V}\left(t_{0} \boldsymbol{\beta}-\mathbf{y}_{1}-\cdots-\mathbf{y}_{n-1}\right) \int_{Q_{R}} e^{-i \phi} d \mathbf{x}_{0} .
\end{aligned}
$$

4. Computing $\phi$ Expressing the phase function $\phi$ in terms of the new variables gives:

$$
\begin{aligned}
\phi= & t_{0} \sum_{j=1}^{n} \omega_{j}\left(\frac{1}{n} x_{0}^{\|}+l_{j}^{\|}\left(y^{\|}-\beta t_{0} \omega\right)\right)^{2}+t_{0} \sum_{j=1}^{n} \omega_{j}\left|\frac{1}{n} x_{0}^{\perp}+l_{j}^{\perp}\left(y^{\perp}\right)\right|^{2} \\
& +\beta t_{0}^{2} \sum_{j=1}^{n} \omega_{j}^{2}\left(\frac{1}{n} x_{0}^{\|}+l_{j}^{\|}\left(y^{\|}-\beta t_{0} \omega\right)\right)+\frac{\beta^{2}}{3} t_{0}^{3} \sum_{j=1}^{n} \omega_{j}^{3} .
\end{aligned}
$$

Expanding and completing squares, we get

$$
\phi=\frac{t_{0}}{n^{2}}\left|\mathbf{x}_{0}+P\left(\beta t_{0}, \omega, y\right)\right|^{2}+\psi\left(t_{0}, \omega, y\right),
$$

where $P$ and $\psi$ are polynomials.

5. Integrating over $\mathbf{x}_{0}$ Using the formula

$$
\lim _{R \rightarrow \infty} \int_{-R}^{R} e^{-i s^{2}} d s=e^{-i \pi / 4} \sqrt{\pi}
$$

and the expression (3.19) for the phase function $\phi$, we obtain

$$
\lim _{R \rightarrow \infty} \int_{Q_{R}} e^{-i \phi} d \mathbf{x}_{0}=e^{-i 3 \pi / 4}\left(\frac{n \sqrt{\pi}}{\sqrt{t_{0}}}\right)^{3} e^{-i \psi\left(t_{0}, \omega, y\right)} .
$$

Due to the fast decay of the integrand in (3.18) in the variables $t_{0}, \mathbf{y}_{1}, \ldots, \mathbf{y}_{n-1}$, one can pass to the limit as $R \rightarrow \infty$, which gives the required integral representation (3.13) with

$$
\begin{aligned}
f(t) & =i^{n} \pi^{-3(n-1) / 2} 2^{-3 n / 2} e^{-i 3 \pi / 4} f_{0}(t) \\
f_{0}(t) & =\int_{\Omega} d \omega \int_{\mathbb{R}^{3}} d \mathbf{y}_{1} \ldots \int_{\mathbb{R}^{3}} d \mathbf{y}_{n-1} \widehat{V}\left(\mathbf{y}_{1}\right) \ldots \widehat{V}\left(\mathbf{y}_{n-1}\right) \widehat{V}\left(t_{0} \boldsymbol{\beta}-\mathbf{y}_{1}-\cdots-\mathbf{y}_{n-1}\right) e^{-i \psi(t, \omega, y)}
\end{aligned}
$$

As $\widehat{V}$ belongs to the Schwartz class on $\mathbb{R}^{3}$, the function $f_{0}$ belongs to the Schwartz class on $\mathbb{R}$.

\section{Formulae (1.11) for the coefficients $Q_{k}$}

1. Notation. For $\varepsilon>0$, denote $\mathbb{C}_{\varepsilon}=\{z \in \mathbb{C} \mid \varepsilon<\arg z<\pi-\varepsilon\}$. Choose $N>0$ big enough so that the ball $\left\{\mathbf{x} \in \mathbb{R}^{3}|| \mathbf{x} \mid<N\right\}$ contains the support of $V$. Let $G_{0} \in C_{0}^{\infty}\left(\mathbb{R}^{3}\right)$ be a function such that $G_{0}(\mathbf{x})=\langle\boldsymbol{\beta}, \mathbf{x}\rangle$ for all $\mathbf{x},|\mathbf{x}| \leq N$. Denote $G(\mathbf{x})=\langle\boldsymbol{\beta}, \mathbf{x}\rangle-G_{0}(\mathbf{x})$. By this definition, $\operatorname{supp} V \cap \operatorname{supp} G=\emptyset$.

2. Strategy. 
Consider the operators

$$
\widetilde{H}_{0}=-\Delta+G_{0}, \quad \widetilde{H}=-\Delta+G_{0}+V .
$$

Similarly to (1.2), we define the perturbation determinants $\Delta_{\widetilde{H}_{0} / T_{0}}(z)$ and $\Delta_{\widetilde{H} / T_{0}}(z)$ (recall that we have denoted $T_{0}=-\Delta$ ). The logarithms of these determinants admit asymptotic expansions of the type (1.7), (1.8) (with $G_{0}$ and $G_{0}+V$ respectively instead of $V$ ). Subtracting these expansions one from another, we get

$$
\log \Delta_{\widetilde{H} / T_{0}}(z)-\log \Delta_{\widetilde{H}_{0} / T_{0}}(z) \sim i Q_{-1} \sqrt{z}+C+\sum_{k=0}^{\infty} \frac{i Q_{k}}{z^{k} \sqrt{z}}, \quad|z| \rightarrow \infty, \quad z \in \mathbb{C}_{+},
$$

where the coefficients $Q_{k}$ are the same as in (1.11):

$$
Q_{k}=\int_{\mathbb{R}^{3}}\left(F_{k}\left[G_{0}(\mathbf{x})+V(\mathbf{x})\right]-F_{k}\left[G_{0}(\mathbf{x})\right]\right) d \mathbf{x}=\int_{\mathbb{R}^{3}}\left(F_{k}[\langle\boldsymbol{\beta}, \mathbf{x}\rangle+V(\mathbf{x})]-F_{k}[\langle\boldsymbol{\beta}, \mathbf{x}\rangle]\right) d \mathbf{x} .
$$

In this section, our aim is to prove that for any $\varepsilon>0$, any $n>0$ and some constant $C \in \mathbb{C}$,

$$
\log \Delta_{H / H_{0}}(z)-\left(\log \Delta_{\widetilde{H} / T_{0}}(z)-\log \Delta_{\widetilde{H}_{0} / T_{0}}(z)\right)=C+O\left(|z|^{-n}\right), \quad|z| \rightarrow \infty, \quad z \in \mathbb{C}_{\varepsilon} .
$$

From (4.1) and (4.2) we get the expansion (1.10) with the coefficients (1.11) in the sector $\mathbb{C}_{\varepsilon}$. By the uniqueness of the coefficients of an asymptotic expansion, get formulae (1.11) for the coefficients $Q_{k}$ of the expansion (1.10).

3. Proof of (4.2). Using (1.5), we see that the derivative of the 1.h.s. of (4.2) with respect to $z$ equals to

$$
\operatorname{Tr}\left[\left((H-z)^{-1}-\left(H_{0}-z\right)^{-1}\right)-\left((\widetilde{H}-z)^{-1}-\left(\widetilde{H}_{0}-z\right)^{-1}\right)\right] .
$$

Instead of proving (4.2), we shall prove a stronger statement:

Lemma 4.1. For any $\varepsilon>0$ and any $n>0$,

$$
\left\|\left((H-z)^{-1}-\left(H_{0}-z\right)^{-1}\right)-\left((\widetilde{H}-z)^{-1}-\left(\widetilde{H}_{0}-z\right)^{-1}\right)\right\|_{\mathfrak{S}_{1}}=O\left(|z|^{-n}\right), \quad|z| \rightarrow \infty, \quad z \in \mathbb{C}_{\varepsilon} .
$$

Before proving Lemma 4.1, let us prove an auxiliary statement. Denote

$$
\widetilde{R}_{0}(z)=\left(\widetilde{H}_{0}-z\right)^{-1} .
$$

Lemma 4.2. Let $\varphi_{1}, \varphi_{2} \in C_{0}^{\infty}\left(\mathbb{R}^{3}\right)$ and suppose that $\varphi_{1} \varphi_{2} \equiv 0$. Then for any $\varepsilon>0$ and any $n>0$,

$$
\left\|\varphi_{1} \widetilde{R}_{0}(z) \varphi_{2}\right\|_{\mathfrak{S}_{2}}=O\left(|z|^{-n}\right), \quad|z| \rightarrow \infty, \quad z \in \mathbb{C}_{\varepsilon}
$$

Proof. Throughout the proof, $\varepsilon>0$ is an arbitrary fixed number and $z \in \mathbb{C}_{\varepsilon}$. It is straightforward to see that $\varphi_{1}\left(T_{0}-z\right)^{-1} \varphi_{2} \in \mathfrak{S}_{2}$ and therefore $\varphi_{1} \widetilde{R}_{0}(z) \varphi_{2} \in \mathfrak{S}_{2}$. From here one easily obtains that

$$
\left\|\varphi_{1} \widetilde{R}_{0}(z) \varphi_{2}\right\|_{\mathfrak{S}_{2}}=O(1), \quad|z| \rightarrow \infty
$$

Next, we will prove (4.4) by induction in $n$ (more precisely, by induction in $2 n$ ). Assume that (4.4) holds true for some value of $n$. One has:

$$
\begin{aligned}
\varphi_{1} \widetilde{R}_{0}(z) \varphi_{2}=\varphi_{1}\left(\widetilde{R}_{0}(z) \varphi_{2}-\varphi_{2} \widetilde{R}_{0}(z)\right) & =\varphi_{1} \widetilde{R}_{0}(z)\left(\varphi_{2} \widetilde{H}_{0}-\widetilde{H}_{0} \varphi_{2}\right) \widetilde{R}_{0}(z) \\
= & \varphi_{1} \widetilde{R}_{0}(z)\left(\Delta \varphi_{2}\right) \widetilde{R}_{0}(z)+\sum_{j=1}^{3} \varphi_{1} \widetilde{R}_{0}(z)\left(\frac{\partial \varphi_{2}}{\partial x_{j}}\right) D_{j} \widetilde{R}_{0}(z),
\end{aligned}
$$


where $D_{j}=\frac{\partial}{\partial x_{j}}$. It is easy to see that

$$
\left\|D_{j} \widetilde{R}_{0}(z)\right\|=O\left(|z|^{-1 / 2}\right), \quad\left\|\widetilde{R}_{0}(z)\right\|=O\left(|z|^{-1}\right), \quad \text { as }|z| \rightarrow \infty
$$

and so (4.5) gives:

$$
\begin{aligned}
& \left\|\varphi_{1} \widetilde{R}_{0}(z) \varphi_{2}\right\|_{\mathfrak{S}_{2}} \leq\left\|\varphi_{1} \widetilde{R}_{0}(z)\left(\Delta \varphi_{2}\right)\right\|_{\mathfrak{S}_{2}} O\left(|z|^{-1}\right) \\
& \quad+\left\|\varphi_{1} \widetilde{R}_{0}(z)\left(\frac{\partial \varphi_{2}}{\partial x_{j}}\right)\right\|_{\mathfrak{S}_{2}} O\left(|z|^{-1 / 2}\right)=O\left(|z|^{-n-\frac{1}{2}}\right), \quad|z| \rightarrow \infty
\end{aligned}
$$

This completes the proof.

Proof of Lemma 4.1. Throughout the proof, $\varepsilon>0$ is an arbitrary fixed number and $z \in \mathbb{C}_{\varepsilon}$. As above, we use the notation $\widetilde{R}_{0}(z)=\left(\widetilde{H}_{0}-z\right)^{-1}$.

1. First recall that (cf. (3.6))

$$
\varphi R_{0}(z) \in \mathfrak{S}_{2}, \quad \varphi \widetilde{R}_{0}(z) \in \mathfrak{S}_{2}, \quad \forall \varphi \in C_{0}^{\infty}\left(\mathbb{R}^{3}\right) .
$$

By a standard argument, it follows that the Hilbert-Schmidt norms of the above operators tend to zero as $|z| \rightarrow \infty$. Thus, one can choose $M>0$ big enough so that

$$
\left\|V R_{0}(z)\right\|_{\mathfrak{S}_{2}} \leq 1 / 2, \quad\left\|V \widetilde{R}_{0}(z)\right\|_{\mathfrak{S}_{2}} \leq 1 / 2, \quad \forall z \in \mathbb{C}_{\varepsilon}, \quad|z| \geq M
$$

2. By (4.8), for $|z| \geq M$ the operator on 1.h.s. of (4.3) can be presented as the sum of convergent series:

$$
\begin{aligned}
& \left((H-z)^{-1}-\left(H_{0}-z\right)^{-1}\right)-\left((\widetilde{H}-z)^{-1}-\left(\widetilde{H}_{0}-z\right)^{-1}\right) \\
& =\sum_{k=1}^{\infty}(-1)^{k} R_{0}(z)\left(V R_{0}(z)\right)^{k}-(-1)^{k} \widetilde{R}_{0}(z)\left(V \widetilde{R}_{0}(z)\right)^{k} .
\end{aligned}
$$

First let us estimate the norm of the remainder term in (4.9):

$$
\sum_{k=n+1}^{\infty}\left\|R_{0}(z)\left(V R_{0}(z)\right)^{k}\right\|_{\mathfrak{S}_{1}} \leq \sum_{k=n+1}^{\infty}\left\|R_{0}(z)\right\|\left\|V R_{0}(z)\right\|_{\mathfrak{S}_{2}}^{2}\left\|V R_{0}(z)\right\|^{k-2}=O\left(|z|^{-n+1}\right), \quad|z| \rightarrow \infty .
$$

In the same way,

$$
\sum_{k=n+1}^{\infty}\left\|\widetilde{R}_{0}(z)\left(V \widetilde{R}_{0}(z)\right)^{k}\right\|_{\mathfrak{S}_{1}}=O\left(|z|^{-n+1}\right), \quad|z| \rightarrow \infty .
$$

Thus, it suffices to prove that for any $k \in \mathbb{N}$ and any $n>0$,

$$
\left\|R_{0}(z)\left(V R_{0}(z)\right)^{k}-\widetilde{R}_{0}(z)\left(V \widetilde{R}_{0}(z)\right)^{k}\right\|_{\mathfrak{S}_{1}}=O\left(|z|^{-n}\right), \quad|z| \rightarrow \infty .
$$

3. One has

$$
\begin{aligned}
& R_{0}(z)\left(V R_{0}(z)\right)^{k}-\widetilde{R}_{0}(z)\left(V \widetilde{R}_{0}(z)\right)^{k}=\left(R_{0}(z)-\widetilde{R}_{0}(z)\right)\left(V R_{0}(z)\right)^{k} \\
& \left.\quad+\widetilde{R}_{0}(z) V\left(R_{0}(z)-\widetilde{R}_{0}(z)\right)\left(V R_{0}(z)\right)\right)^{k-1}+\cdots+\widetilde{R}_{0}(z)\left(V \widetilde{R}_{0}(z)\right)^{k-1} V\left(R_{0}(z)-\widetilde{R}_{0}(z)\right) .
\end{aligned}
$$


Thus, for $k \geq 2$ the relation (4.10) will follow if we prove that

$$
\left\|V\left(R_{0}(z)-\widetilde{R}_{0}(z)\right)\right\|_{\mathfrak{S}_{2}}=O\left(|z|^{-n}\right), \quad|z| \rightarrow \infty, \quad \forall n>0 .
$$

For $k=1$, we use the following simple trick: find $\varphi \in C_{0}^{\infty}\left(\mathbb{R}^{3}\right)$ such that $\varphi V=V$. Then

$$
\begin{aligned}
& R_{0}(z) V R_{0}(z)-\widetilde{R}_{0}(z) V \widetilde{R}_{0}(z)=\left(R_{0}(z)-\widetilde{R}_{0}(z)\right) V \varphi R_{0}(z) \\
& +\widetilde{R}_{0}(z) \varphi V\left(R_{0}(z)-\widetilde{R}_{0}(z)\right)+\widetilde{R}_{0}(z) \varphi V\left(R_{0}(z)-\widetilde{R}_{0}(z)\right) .
\end{aligned}
$$

By (4.7), we see that for $k=1$ the relation (4.10) will also follow from (4.11).

4. Let us prove (4.11). One has

$$
V\left(R_{0}(z)-\widetilde{R}_{0}(z)\right)=-V \widetilde{R}_{0}(z) G R_{0}(z) .
$$

Performing the computation analogous to (4.5) twice, one obtains:

$$
\begin{aligned}
V \widetilde{R}_{0}(z) G=V \widetilde{R}_{0}(z)(\Delta G) \widetilde{R}_{0}(z)+\sum_{j=1}^{3} V \widetilde{R}_{0}(z)\left(\frac{\partial}{\partial x_{j}} \Delta G\right) D_{j} \widetilde{R}_{0}(z) \\
+\sum_{j, l=1}^{3} V \widetilde{R}_{0}(z)\left(\frac{\partial^{2}}{\partial x_{j} \partial x_{l}} G\right) D_{j} \widetilde{R}_{0}(z) D_{l} \widetilde{R}_{0}(z) .
\end{aligned}
$$

Note that the functions $\Delta G, \frac{\partial}{\partial x_{j}} \Delta G$, and $\frac{\partial^{2}}{\partial x_{j} \partial x_{l}} G$ belong to the class $C_{0}^{\infty}\left(\mathbb{R}^{3}\right)$ and the support of either of them is disjoint with the support of $V$. Recalling (4.4) and (4.6), we obtain that for any $n>0,\left\|V \widetilde{R}_{0}(z) G\right\|_{\mathfrak{S}_{2}}=O\left(|z|^{-n}\right)$. This proves (4.11).

\section{The scattering matrix}

In this section we recall the connection between the spectral shift function and the scattering matrix and, using this connection, prove Lemma 1.3.

1. The spectral representation for $H_{0}$. We will need some facts concerning the spectral decomposition of $H_{0}$, which we denote by $E_{0}(\lambda) \equiv \theta\left(\lambda-H_{0}\right)$. First we recall formulae for $E_{0}(\lambda)$ due to [1]. Let $f \mapsto \widetilde{f}$ be the unitary transformation in $L^{2}\left(\mathbb{R}^{3}\right)$, which can be defined on $L^{1}\left(\mathbb{R}^{3}\right) \cap L^{2}\left(\mathbb{R}^{3}\right)$ by the explicit formula

$$
\widetilde{f}(\mathbf{p})=(2 \pi)^{-1} \int_{\mathbb{R}^{3}} e^{-i\left\langle p^{\perp}, x^{\perp}\right\rangle} \beta^{1 / 3} \operatorname{Ai}\left(\beta^{1 / 3}\left(x^{\|}-p^{\|}\right)\right) f(\mathbf{x}) d \mathbf{x},
$$

where $\mathrm{Ai}$ is the Airy function. This unitary transformation carries over $H_{0}$ into multiplication by $\beta p^{\|}+\left|p^{\perp}\right|^{2}$ in $L^{2}\left(\mathbb{R}^{3}, d \mathbf{p}\right)$ :

$$
\left(H_{0} f\right)^{\sim}(\mathbf{p})=\left(\beta p^{\|}+\left|p^{\perp}\right|^{2}\right) \widetilde{f}(\mathbf{p}) .
$$

Thus, for any $f \in L^{2}\left(\mathbb{R}^{3}\right)$, the quadratic form of $E_{0}(\lambda)$ can be presented as

$$
\left(E_{0}(\lambda) f, f\right)=\int_{\beta p^{\|}+\left|p^{\perp}\right|^{2}<\lambda}|\widetilde{f}(\mathbf{p})|^{2} d \mathbf{p}=\int_{\mathbb{R}^{2}} d p^{\perp} \int_{-\infty}^{\left(\lambda-\left|p^{\perp}\right|^{2}\right) / \beta}|\widetilde{f}(\mathbf{p})|^{2} d p^{\|} .
$$


Differentiation with respect to $\lambda$ gives

$$
\frac{d}{d \lambda}\left(E_{0}(\lambda) f, f\right)=(J(\lambda) f, J(\lambda) f), \quad f \in L^{1}\left(\mathbb{R}^{3}\right) \cap L^{2}\left(\mathbb{R}^{3}\right),
$$

where $J(\lambda): L^{1}\left(\mathbb{R}^{3}\right) \cap L^{2}\left(\mathbb{R}^{3}\right) \rightarrow L^{2}\left(\mathbb{R}^{2}\right)$ is given by

$$
(J(\lambda) f)\left(p^{\perp}\right)=(2 \pi)^{-1} \int_{\mathbb{R}^{3}} e^{-i\left\langle p^{\perp}, x^{\perp}\right\rangle} \beta^{-2 / 3} \operatorname{Ai}\left(\beta^{1 / 3} x^{\|}+\beta^{-2 / 3}\left|p^{\perp}\right|^{2}-\beta^{-2 / 3} \lambda\right) f(\mathbf{x}) d \mathbf{x} .
$$

Recall (see (3.1)) that $W_{1}=\sqrt{|V|}$.

Lemma 5.1. For any $\lambda \in \mathbb{R}$, the operator

$$
J(\lambda) W_{1}: L^{2}\left(\mathbb{R}^{3}\right) \rightarrow L^{2}\left(\mathbb{R}^{2}\right)
$$

belongs to the Hilbert-Schmidt class $\mathfrak{S}_{2}$ and is continuous and differentiable in the $\mathfrak{S}_{2}$ norm. One has

$$
\left\|J(\lambda) W_{1}\right\|_{\mathfrak{S}_{2}}=O\left(|\lambda|^{-N}\right), \quad \lambda \rightarrow-\infty, \quad \forall N>0 .
$$

Proof. The proof is a direct estimate of the Hilbert-Schmidt norm:

$$
\begin{aligned}
\left\|J(\lambda) W_{1}\right\|_{\mathfrak{S}_{2}}^{2} & =(2 \pi)^{-2} \int_{\mathbb{R}^{3}} d \mathbf{x} \int_{\mathbb{R}^{2}} d p^{\perp} \beta^{-4 / 3}\left|\operatorname{Ai}\left(\beta^{1 / 3} x^{\|}+\beta^{-2 / 3}\left|p^{\perp}\right|^{2}-\beta^{-2 / 3} \lambda\right)\right|^{2}|V(\mathbf{x})| ; \\
\left\|\frac{d}{d \lambda} J(\lambda) W_{1}\right\|_{\mathfrak{S}_{2}}^{2} & =(2 \pi)^{-2} \int_{\mathbb{R}^{3}} d \mathbf{x} \int_{\mathbb{R}^{2}} d p^{\perp} \beta^{-8 / 3}\left|\operatorname{Ai}^{\prime}\left(\beta^{1 / 3} x^{\|}+\beta^{-2 / 3}\left|p^{\perp}\right|^{2}-\beta^{-2 / 3} \lambda\right)\right|^{2}|V(\mathbf{x})| .
\end{aligned}
$$

Due to the superexponential decay of $\operatorname{Ai}(t)$ and $\operatorname{Ai}^{\prime}(t)$ as $t \rightarrow+\infty$, the integrals converge and the estimate (5.4) holds true.

2. The scattering matrix. The wave operators $W_{ \pm}=W_{ \pm}\left(H, H_{0}\right)$ for the pair (1.1) exist and are complete (see $[1,9]$ ) and thus the scattering operator $S=W_{+}^{*} W_{-}$is well defined and unitary. The operators $H_{0}$ and $S$ commute and thus are simultaneously diagonalizable:

$$
L^{2}\left(\mathbb{R}^{3}\right)=\int_{\mathbb{R}}^{\oplus} \mathcal{H}(\lambda) d \lambda, \quad H_{0}=\int_{\mathbb{R}}^{\oplus} \lambda I_{\lambda} d \lambda, \quad S=\int_{\mathbb{R}}^{\oplus} S(\lambda) d \lambda
$$

here $I_{\lambda}$ is the identity in the fiber space $\mathcal{H}(\lambda)$ and $S(\lambda)$ is the scattering matrix for the pair (1.1). According to the diagonalization formulae (5.1), (5.2), all the fiber spaces $\mathcal{H}(\lambda)$ coincide with $L^{2}\left(\mathbb{R}^{2}\right)$. The stationary representation for the scattering matrix reads (see e.g. [17]):

$$
S(\lambda)=I-2 \pi i\left(J(\lambda) W_{2}\right)(I+T(\lambda+i 0))^{-1}\left(J(\lambda) W_{1}\right)^{*}, \quad \lambda \in \mathbb{R}
$$

note that the operator $I+T(\lambda+i 0)$ is invertible for all $\lambda \in \mathbb{R}$ (see the proof of Lemma 1.1(iv)).

By a standard argument (cf. the proof of Lemma 1.1(iii) in the Appendix), Lemma 5.1 implies that $T(\lambda+i 0)$ is continuous in $\lambda \in \mathbb{R}$ in the operator norm. Therefore, we obtain that $S(\lambda)-I$ is continuous in $\lambda$ in the trace norm. Thus, the function $\operatorname{det} S(\lambda)$ is continuous in $\lambda$.

3. The Birman-Krein formula. According to the Birman-Krein formula [2],

$$
\operatorname{det} S(\lambda)=e^{-2 \pi i \xi(\lambda)}, \quad \lambda \in \mathbb{R} .
$$

As both $\xi(\lambda)$ and $\operatorname{det} S(\lambda)$ are continuous in $\lambda$, formula (5.6) determines $\xi(\lambda)$ up to a constant integer term.

From Lemma 3.1, Lemma 5.1 and the stationary representation (5.5) we immediately obtain the estimate

$$
\left\|S(\lambda)-I_{\lambda}\right\|_{\mathfrak{S}_{1}}=O\left(|\lambda|^{-N}\right), \quad \lambda \rightarrow-\infty, \quad \forall N>0 .
$$

Thus, by (5.6), the spectral shift function $\xi(\lambda)$ satisfies (1.15). This proves Lemma 1.3. 


\section{Appendix: Proof of Lemma 1.1}

The proof is a combination of standard results and techniques of scattering theory for trace class and smooth perturbations.

1. Let us prove the statement (i). The equality in (1.3) is a matter of direct calculation. In order to check that the operator in (1.3) belongs to the trace class, first recall the inclusion (3.6). Applying the resolvent identity yields

$$
W_{1}(H-z)^{-1} \in \mathfrak{S}_{2}, \quad \forall z \in \mathbb{C}_{+},
$$

which gives the required result.

2. Let us prove the statements (ii), (iii). For $z \in \mathbb{C}_{+}$one has

$$
T(z)-T(-i)=(z+i) W_{1} R_{0}(z) R_{0}(-i) W_{2} \in \mathfrak{S}_{1}
$$

by (3.6). Denote for brevity $R(z)=(H-z)^{-1}$. A straightforward computation yields

$$
\begin{aligned}
\Delta_{H / H_{0}}(z) & =\operatorname{det}(I-(z+i) R(-i))\left(I+(z+i) R_{0}(z)\right) \\
& =\operatorname{det}\left[I+(z+i)\left(R_{0}(z)-R(-i)-(z+i) R(-i) R_{0}(z)\right)\right] \\
& =\operatorname{det}\left[I+(z+i) R(-i) V R_{0}(z)\right] \quad(\text { using } \operatorname{det}(I+A B)=\operatorname{det}(I+B A)) \\
& =\operatorname{det}\left[I+(z+i) W_{1} R_{0}(z) R(-i) W_{2}\right]=\operatorname{det}\left[I+W_{1}\left(R_{0}(z)-R(-i)-R_{0}(z) V R(-i)\right) W_{2}\right] \\
& =\operatorname{det}\left(I+W_{1} R_{0}(z) W_{2}\right)\left(I-W_{1} R(-i) W_{2}\right)=\operatorname{det}(I+T(z))(I+T(-i))^{-1} \\
& =\operatorname{det}\left[I+(T(z)-T(-i))(I+T(-i))^{-1}\right] .
\end{aligned}
$$

The operator $T(z)-T(-i)$ is analytic in $z \in \mathbb{C}_{+}$, which proves that $\Delta_{H / H_{0}}(z)$ is analytic in $\mathbb{C}_{+}$. In order to prove the rest of the statements, below we to check that for any finite open interval $\delta \subset \mathbb{R}$ and any $\lambda \in \delta$, the limit $T(\lambda+i 0)-T(-i)$ exists in the trace norm and is locally Hölder continuous in $\lambda \in \delta$ in the trace norm.

Let $E_{0}(\mathbb{R} \backslash \delta)$ be the spectral projection of $H_{0}$, associated to the complement of $\delta$ in $\mathbb{R}$. One has

$$
T(z)-T(-i)=(z+i) \int_{\delta} \frac{\left(J(\lambda) W_{1}\right)^{*}\left(J(\lambda) W_{2}\right)}{(t+i)(t-z)} d t+(z+i) W_{1} R_{0}(z) E_{0}(\mathbb{R} \backslash \delta) R_{0}(-i) W_{2},
$$

where $J(\lambda)$ is defined by (5.3). By Lemma 5.1, the integral in (6.2) has a limit as $z \rightarrow \lambda+i 0$, $\lambda \in \delta$, and this limit is Hölder continuous (with any Hölder exponent $\gamma \in(0,1)$ ). The second term in the r.h.s. of $(6.2)$ is smooth in $z \in \delta$.

3. Let us prove the statement (iv). Recall (6.1):

$$
\Delta_{H / H_{0}}(z)=\operatorname{det}(I+T(z))(I+T(-i))^{-1} .
$$

As $(I+T(-i))$ is bounded and invertible, it is sufficient to check that $(-1)$ is not an eigenvalue of $T(z)$ for $z \in \mathbb{C}_{+}$or for $z=\lambda+i 0, \lambda \in \mathbb{R}$. If $T(z) \psi=-\psi$ for $\psi \in L^{2}\left(\mathbb{R}^{3}\right)$ and $z \in \mathbb{C}_{+}$, then $H \phi=z \phi$, where $\phi=R_{0}(z) W_{2} \psi \in L^{2}\left(\mathbb{R}^{3}\right)$. This contradicts the self-adjointness of $H$.

4. Finally, let us check that $(-1)$ is not an eigenvalue of $T(\lambda+i 0)$ for $\lambda \in \mathbb{R}$. Suppose that

$$
T(\lambda+i 0) \psi=-\psi \quad \text { for } \psi \in L^{2}\left(\mathbb{R}^{3}\right) \quad \text { and } \lambda \in \mathbb{R} .
$$


Let us use a standard argument (due to $\mathrm{S}$. Agmon) to show that $\lambda$ is an eigenvalue of $H$. It is sufficient to prove that

$$
R_{0}(\lambda+i 0) W_{2} \psi \in L^{2}\left(\mathbb{R}^{3}\right),
$$

because (6.3) and (6.4) yield $H\left(R_{0}(\lambda+i 0) W_{2} \psi\right)=\lambda\left(R_{0}(\lambda+i 0) W_{2} \psi\right)$.

In order to prove (6.4), first take the inner product of (6.3) with $W_{2} R_{0}(\lambda+i 0) W_{2} \psi$ and then take the imaginary part of the resulting equation. This gives $J(\lambda) W_{2} \psi=0$ (where $J(\lambda)$ is defined by (5.3)). Next, let $\delta=(\lambda-1, \lambda+1)$. The norm of $R_{0}(\lambda+i 0) W_{2} \psi$ can be computed as follows:

$$
\left\|R_{0}(\lambda+i 0) W_{2} \psi\right\|^{2}=\left\|E_{0}(\mathbb{R} \backslash \delta) R_{0}(\lambda+i 0) W_{2} \psi\right\|^{2}+\int_{\delta} \frac{\left\|J(t) W_{2} \psi\right\|^{2}}{|t-\lambda|^{2}} d t .
$$

The first term in the r.h.s. is finite. The second term can be estimated by using Lemma 5.1:

$$
\left\|J(t) W_{2} \psi\right\|^{2}=\left\|(J(t)-J(\lambda)) W_{2} \psi\right\|^{2} \leq C(\lambda)|t-\lambda|^{2}\|\psi\|^{2},
$$

so the integral converges, the norm is finite, and we get (6.4).

Thus, $\lambda$ is an eigenvalue of $H$. But, by a result of [1], the operator $H$ has no eigenvalues. Therefore, (6.3) is impossible.

\section{Acknowledgments}

The research was supported by the London Mathematical Society grant under Scheme 4 ref. 4535 and by the SFB grant no. 288. The second author is grateful to R. Halburd for useful discussions.

\section{References}

[1] J. E. Avron and I. W. Herbst, Spectral and scattering theory of Schrödinger operators related to the Stark effect, Commun. Math. Phys. 52 (1977), 239-254.

[2] M. Sh. Birman and M. G. Krein, On the theory of wave operators and scattering operators (in Russian), Dokl. Akad. Nauk SSSR 144 (1962), 475-478; English transl. in Soviet Math. Dokl. 3 (1962).

[3] M. Sh. Birman, D. R. Yafaev, The spectral shift function. The papers of M. G. Krein and their further development. (in Russian) Algebra i Analiz 4, no. 5 (1992), 1-44. English translation: St. Petersburg Math. J. 4, no. 5 (1993), 833-870.

[4] V. S. Buslaev, L. D. Faddeev, Formulas for traces for a singular Sturm-Liouville differential operator. Dokl. Akad. Nauk SSSR 132 (1960), 13-16 (Russian). English translation: Soviet Math. Dokl. 1 (1960), 451-454.

[5] Buslaev, V. S. Trace formulas for the Schrödinger operator in a three-dimensional space. (Russian) Dokl. Akad. Nauk SSSR 143 (1962), 1067-1070.

[6] Buslaev, V.S. The trace formulae and certain asymptotic estimates of the kernel of the resolvent for the Schrödinger operator in three-dimensional space. (Russian) (1966) Probl. Math. Phys., No. I, Spectral Theory and Wave Processes (Russian) pp. 82-101 Izdat. Leningrad. Univ., Leningrad 
[7] Y. Colin de Verdière, Une formule de traces pour l'opérateur de Schrödinger dans $\mathbf{R}^{3}$, Ann. Sci. École Norm. Sup. 14, (1981), 27-39.

[8] L. Guillopé, Asymptotique de la phase de diffusion pour l'opérateur de Schrödinger dans $\mathbf{R}^{n}$, Séminaire E.D.P., 1984-1985, Exp. No. V, Ecole Polytechnique, 1985.

[9] I. W. Herbst, Unitary equivalence of Stark Hamiltonians, Math. Z. 155 (1977), 55-70.

[10] M. Hitrik, I. Polterovich, Regularized traces and Taylor expansions for the heat semigroup, preprint September 2001.

[11] E. L. Korotyaev, On the theory of multiparticle scattering in an external electric field. (Russian) Mat. Sb. (N.S.) 132(174) (1987), no. 2, 182-201. translation in Math. USSR-Sb. 60 (1988), no. 1, 177-196.

[12] G. Popov, Asymptotic behaviour of the scattering phase for the Schrödinger operator, C. R. Acad. Bulgare Sci. 35 (1982), no. 7, 885-888.

[13] M. Reed, B. Simon, Methods of modern mathematical physics, vol. 4, Academic Press, 1978.

[14] D. Robert, Asymptotique à grande energie de la phase de diffusion pour un potentiel, Asymptot. Anal. 3 (1991), 301-320.

[15] D. Robert, X. P. Wang Time-delay and spectral density for Stark Hamiltonians. II. Asymptotics of trace formulae. Chinese Ann. Math. Ser. B 12 (1991), no. 3, 358-383.

[16] D. R. Yafaev, Mathematical scattering theory. General theory, Amer. Math. Soc., Providence, RI, 1992.

[17] K. Yajima, Spectral and scattering theory for Schrödinger operators with Stark effect. II. J. Fac. Sci. Univ. Tokyo Sect. IA Math. 28 (1981), no. 1, 1-15. 